\title{
Analyzing patients' EEG energy for brain death determination based on Dynamic 2T-EMD
}

\author{
Yao Miao, Dongsheng Wang, Gaochao Cui, Li Zhu, Jianting Cao \\ Saitama Institute of Technology, Fusaiji 1690, Fukaya, Saitama, Japan \\ e6006gxp@sit.ac.jp \\ Saitama Institute of Technology, Fusaiji 1690, Fukaya, Saitama, Japan \\ d5001emw@sit.ac.jp \\ Saitama Institute of Technology, Fusaiji 1690, Fukaya, Saitama, Japan \\ e4001hbx@sit.ac.jp \\ School of Information Science and Engineering, Xiamen University, Xiamen, Fujian, China \\ zhulibrain@gmail.com \\ Saitama Institute of Technology, Fusaiji 1690, Fukaya, Saitama, Japan \\ cao@sit.ac.jp
}

\begin{abstract}
EEG (electroencephalography) energy is an important evaluation indicator in brain death determination based on EEG analysis. In related works, the static EEG energy value can be discovered using EMD (empirical mode decomposition), MEMD (multivariate empirical mode decomposition) and 2T-EMD (turning tangent empirical mode decomposition) for EEG-based coma and quasi-brain-death analysis. However such methods are not time-varying and feasible. In this paper, we firstly propose the Dynamic 2T-EMD algorithm to evaluate the dynamic patients' EEG energy variation by the means of time window and time step method. With the time window sliding along the time axis in a time step, EEG energy of corresponding time step is computed and stored. The proposed algorithm is applied to analyze 19 cases of coma patients' EEG and 17 cases of quasi-brain-death patients' EEG. Two typical patients in coma and quasi-brain-death state and one special case who was from coma to quasi-brain-death have been taken as examples to give the algorithm performance. Results show that EEG energy in coma state are obviously higher than that in quasi-brain-death state, and even present the EEG energy change trend of every case, which can prevent loss of information and wrong analysis results caused by noise interference and provide scientific basis for doctors to evaluate patients' consciousness levels in brain death determination. The proposed algorithm will be very helpful to develop the real time brain death diagnostic system.
\end{abstract}

\section{Keywords}

EEG energy analysis; Dynamic 2T-EMD; Brain death determination

\section{Academic Discipline And Sub-Disciplines}

Signal processing; Computer Science; Neuronscience

\section{SUBJECT CLASSIFICATION}

Signal Processing; Signal Analysis

\section{TYPE (METHOD/APPROACH)}

Algorithms Analysis; Experimentation; Clinic

\section{INTRODUCTION}

Brain death is strictly defined that the complete, irreversible and permanent loss of brain and brain-stem function (Becheer et al., 1968; Wijdicks EFM et al., 2002). Based on the definition, more than 80 countries in the world have established brain death determination standards respectively. Previous researches on brain death determination were mainly concentrated in the field of clinic medicine. It is on the rise that objective scientific basis and indicators from the perspective of neuro dynamics are provided for the brain death determination. More specifically, researches of brain death determination based on EEG analysis are progressing rapidly.

Several EEG analysis algorithms such as ICA (independent component analysis) (A. Hyvarinen et al., 1997; L. Li et al., 2008; Gennady G. Knyazev et al., 2011), EMD (N. Huang et al., 1998), MEMD (N. Rehman et al., 2010), and 2T-EMD (Julien Fleureau et al., 2011) are applied to analyze coma and quasi-brain-death patients' EEG. ICA was applied in patients' EEG analysis because of its strong ability of denoising and component extraction (J. Cao et al., 2003; Z. Chen et al., 2008), but it's lack of rigorous basis for the determination of the patient whose brain activity components were not extracted. EMD based algorithms, as fully data driven algorithms, could analyze nonlinear and nonstationary signals and compute energy of signal at any time to avoid the loss of signal information. So EEG energy indicator was introduced to analyze quantitatively patients' EEG (E. Niedermeyer et al., 1991), and EMD based static algorithms such as EMD, MEMD, and 2T-EMD were all applied to process EEG and also compute EEG energy (M. Tomasz et al., 2010; Q. Shi et al., 2011). But a static EEG value was only obtained, which can't reflect dynamically patients' status. 
In this paper, we firstly propose the Dynamic 2T-EMD to analyze dynamically coma and quasi-brain-death patients' EEG. The algorithm is developed by modification of the existing $2 T$-EMD on the time axis, using the time window and time step. With the time window sliding along the time axis, the EEG energy in corresponding to time window is computed and stored. We applied the Dynamic 2T-EMD to analyze 36 cases of patients' EEG energy, and focused on analyzing the three cases, respectively one coma patient's EEG, one quasi-brain-death patient's EEG and one patient's EEG from coma to quasibrain-death. And then 36 cases of average EEG energy value of 6 channels for entire recording time are computed. The results show that the EEG energy in coma state is obviously higher than the EEG energy in quasi-brain-death state. More importantly, because of the non-stationarity feature of EEG, the results obtained can reflect dynamically patients' status of the whole period of measurement time and avoid the loss of information and wrong results caused by noise interference, which can provide doctors with the objective and scientific criterian for the clinical diagnosis of brain death determination. Furthermore, the developed algorithm is extremely important to the real time brain death diagnostic system.

\section{THE DYNAMIC 2T-EMD ALGORITHM}

\subsection{T-EMD algorithm}

2T-EMD, belonging to EMD based static algorithms, is a fully data-driven algorithm for mono- and multivariate signals processing. Specifically, 2 T-EMD could decompose a given signal $s$ into a set of IMFs (intrinsic mode functions) $\sum_{i=1}^{n} I M F_{i}$ and a monotonic residual signal $r(n)$, shown in formula (1).

$$
s=\sum_{i=1}^{n} I M F_{i}+r(n)
$$

As 2T-EMD can decompose both mono- and multivariate signals directly, the key of 2T-EMD is the computation of signal mean trend, which is obtained by averaging two envelopes: a first envelope interpolates the even indexed barycenters which include signal borders, and a second envelope interpolates the odd indexed barycenters which also include signal borders (Julien Fleureau et al., 2011). Let $s$ be a class $C^{1}$ function in $R^{D}$ domain and differentiable with a continuous first derivative. The sifting procesure of computating the signal mean trend is briefly illustrated as below.

(1) Defined a time series $\vec{T}(s)$ as the tangent vector to $s$ and express as $\vec{T}(s) \rightarrow\left[1, \frac{d s}{d t}(t)\right]$.

(2) Defined $\alpha(s)$ as the Euclidean inner products of $R^{D+1}$ and express as $\alpha(s) \rightarrow \lim _{h \rightarrow 0}\left\langle\vec{T}_{s}(t-h), \vec{T}_{s}(t+h)\right\rangle$. And due to the continuity of Euclidean inner product, so $\alpha(s)$ can be expressed as $\forall t \in R, \alpha_{s}(t)=\lim _{h \rightarrow 0}\left\langle\vec{T}_{s}(t-h), \vec{T}_{s}(t+h)\right\rangle$.

(3) Since $s$ is a class $C^{1}$ function, we can get $\alpha(s)=\left\|\vec{T}_{s}\right\|^{2}=1+\left\|\frac{d s}{d t}(t)\right\|^{2}$. Where $\|\cdot\|$ refers to the Euclidean norm of both $R^{D}$ and $R^{D+1}$.

(4) Oscillation extremum of function $s(t)$ is defined as the local minimum of function $\beta_{s}(t): \beta_{s}(t)=\left\|\frac{d s}{d t}(t)\right\|^{2}$.

(5) Take two consecutive oscillation extrema, respectively $P_{1}=\left[t_{1}, s\left(t_{1}\right)\right]^{T}$ and $P_{2}=\left[t_{2}, s\left(t_{2}\right)\right]^{T}$, thereby $M_{P_{1}-P_{2}}$, the barycenter of the associated elementary oscillation is defined as $M_{P_{1}-P_{2}}=\left[\frac{t_{1}+t_{2}}{2}, \frac{1}{t_{1}-t_{2}} \int_{t_{2}}^{t_{1}} s(t) d t\right]^{T}$.

(6) Then the signal mean vector $\vec{e}(t)$ can be obtained according to the definition above.

\subsection{Dynamic 2T-EMD algorithm}
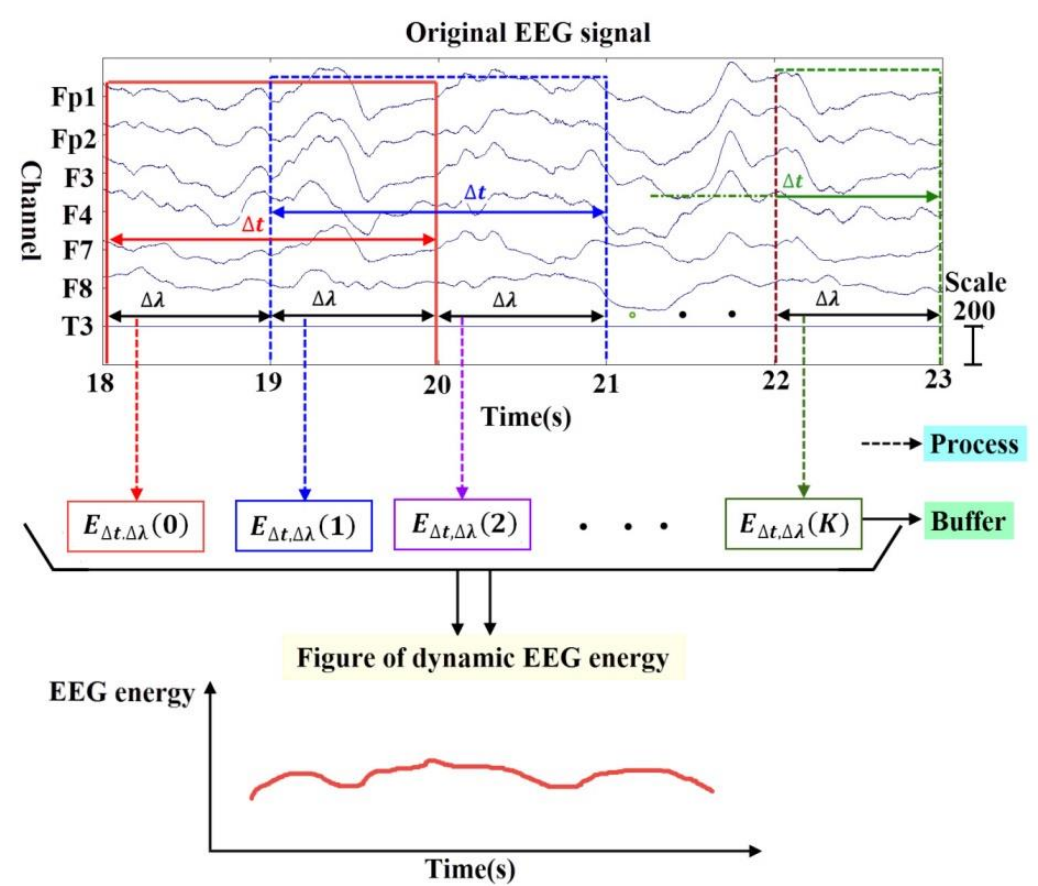

Fig. 1 Schematic diagram of Dynamic 2T-EMD 
Dynamic 2T-EMD is developed by extending $2 \mathrm{~T}$-EMD based on $2 \mathrm{~T}$-EMD that is with excellent static EEG energy computational performance. As is shown in Figure 1, In the Dynamic 2T-EMD algorithm, a time window that the width is $\Delta t$ and a time step with the width $\Delta \lambda$ are introduced, where $\Delta t$ and $\Delta \lambda$ are controllable parameters. With the time window sliding along the time axis in a time step, a time step of EEG is processed and value is stored, then repeat the steps above. And finally we obtain a collection of ordered data. More specifically, for a multivariate signal with $n$ components $\{\vec{s}(k \cdot \Delta t)\}_{k=0}^{K}=\{\vec{s}(0 \cdot \Delta t), \vec{s}(1 \cdot \Delta t), \cdots, \vec{s}(K \cdot \Delta t)\}$ from $T_{1}$ to $T_{2}$, where $T_{2}=T_{1}+K \cdot \Delta t$. The decomposition process of Dynamic 2T-EMD and the flow chart of decomposition process for Dynamic 2T-EMD are respectively as shown in Figure 1 and Figure 2.

(1) Initialize the number of iteration $j=1$, the number of IMF $i=1$, , and the number of time step $k=0$; and set $\vec{r}_{i}(k$. $\Delta t)=\{\vec{s}(k \cdot \Delta t)\}_{k=0}^{K}, \vec{h}_{i, j-1}(k \cdot \Delta t)=\vec{r}_{i}(k \cdot \Delta t)$.

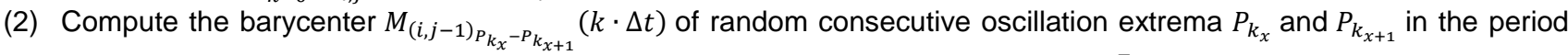
of $\mathrm{k} \cdot \Delta \mathrm{t}$, that is $M_{(i, j-1)}(k \cdot \Delta t)=\left[\frac{k_{P_{x}} \cdot \Delta t+k_{x+1} \cdot \Delta t}{2}, \frac{1}{k_{k_{x}} \cdot \Delta t-k_{x+1} \cdot \Delta t} \int_{k_{x+1} \cdot \Delta t}^{k_{x} \cdot \Delta t} \vec{h}_{i, j-1}(t) d t\right]^{T}$.

(3) Obtain the signal mean trend $\vec{e}_{i, j-1}(k \cdot \Delta t)$ by interpolating between oscillation barycenters of $\vec{h}_{i, j-1}(k \cdot \Delta t)$.

(4) Substracting $\vec{e}_{i, j-1}(k \cdot \Delta t)$ from the given signals $\vec{h}_{i, j-1}(k \cdot \Delta t)$, we define $\vec{h}_{i, j}(k \cdot \Delta t)=\vec{h}_{i, j-1}(k \cdot \Delta t)-\vec{e}_{i, j-1}(k \cdot \Delta t)$. If $\vec{h}_{i, j}(k \cdot \Delta t)$ obey the sifting stop criteria, defining $\overrightarrow{I M F}_{i}(k \cdot \Delta t)=\vec{h}_{i, j}(k \cdot \Delta t)$, otherwise repeat the iteration steps from (2) to (4). It is worth noting that the stop criteria is using the Cauchy-like criteria, more precisely, let $d_{i, j}$ be the $i-t h$ IMF computed at the $j-t h$ iteration of the sifting process, then the sifting criteria is for instance $90 \%$ of values $\left\|\frac{d_{i, j+1}(t)-d_{i, j}(t)}{d_{i, j}(t)}\right\|$ are lower than $10^{-2}$.

(5) Define $\vec{r}_{i}(k \cdot \Delta t)=\vec{r}_{i}(k \cdot \Delta t)-\overrightarrow{I M F}_{i}(k \cdot \Delta t)$, if the result signal is monotonous, we can get the decomposition result of signal during $k \cdot \Delta t$, that is $\vec{s}(k \cdot \Delta t)=\sum_{i=1}^{N} \overrightarrow{I M F}_{i}(k \cdot \Delta t)+\vec{r}_{N}(k \cdot \Delta t)$, otherwise repeat steps from (2) to (5).

(6) Determine whether the elapsed time $k \cdot \Delta t$ exceeds the end time $T_{2}$, if it does, the process goes to end and the final decomposition result $\{\vec{s}(k \cdot \Delta t)\}_{k=0}^{K}=\left\{\sum_{i=1}^{N} \overrightarrow{I M F}_{i}(k \cdot \Delta t)+\vec{r}_{N}(k \cdot \Delta t)\right\}_{k=0}^{K}$; If it doesn't, moving the time window and repeat steps from (2) to (6).

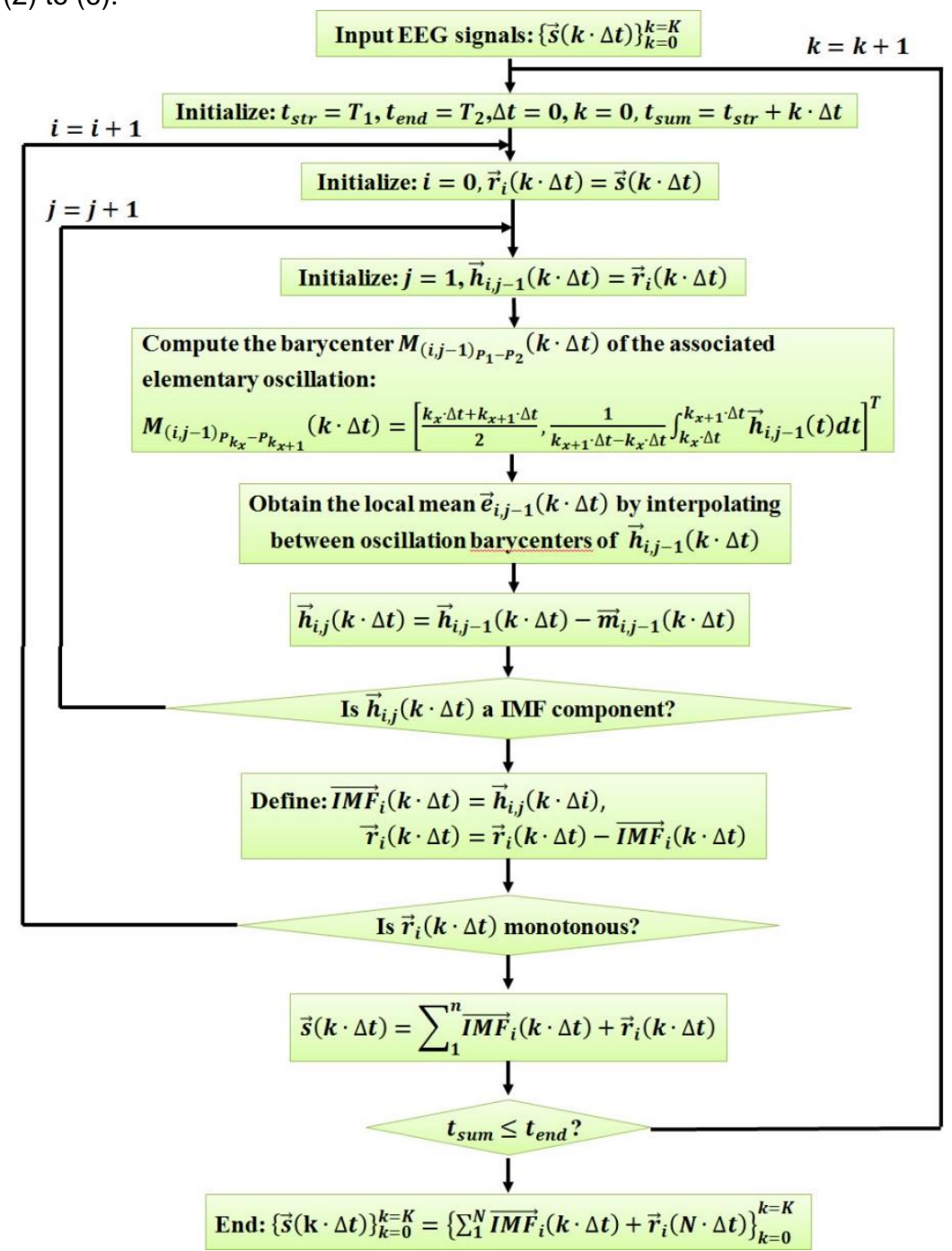

Fig. 2 Flow chart of Dynamic 2T-EMD 


\section{EXPERIMENTS AND RESULTS}

\subsection{Experiments}

In this paper, we apply Dynamic 2T-EMD to process 36 cases of coma and quasi-brain-death patients' EEG (19 coma, 17 quasi-brain-death). The 36 cases of patients' EEG was recorded from 35 patients (male:20, female:15), with age ranging from 17 to 85 years old. It is noted that 36 cases of patients' EEG were recorded in EEG preliminary examination in a Chinese hospital in Shanghai from June 2004 to March 2006, with the permission of patients' families. Considering the specificity of patients' symptoms, the measure of placing the high-purity electrodes on the forehead to record EEG was used in the EEG preliminary examination (J. Cao et al., 2006). The portable electroencephalograph with NEUROSCAN ESI-64 system was applied, where 7 electrodes were placed on the forehead of patients, respectively 6 exploring electrodes (Fp1, Fp2, F3, F4, F7, F8) and 1 ground electrode (GND), and 2 electrodes (A1, A2) as reference electrodes were placed on earlobes. The sampling rate of EEG was $1000 \mathrm{~Hz}$ and the electrode resistance was lower than $8 \mathrm{k} \Omega$, shown in Figure 3. Moreover, EEG energy is defined as that the power spectrum within the frequency band multiplied by recorded EEG time. When there are obvious periodic rhythms in the EEG signal, that is to say, there exists brain activity, the corresponding EEG energy is higher.

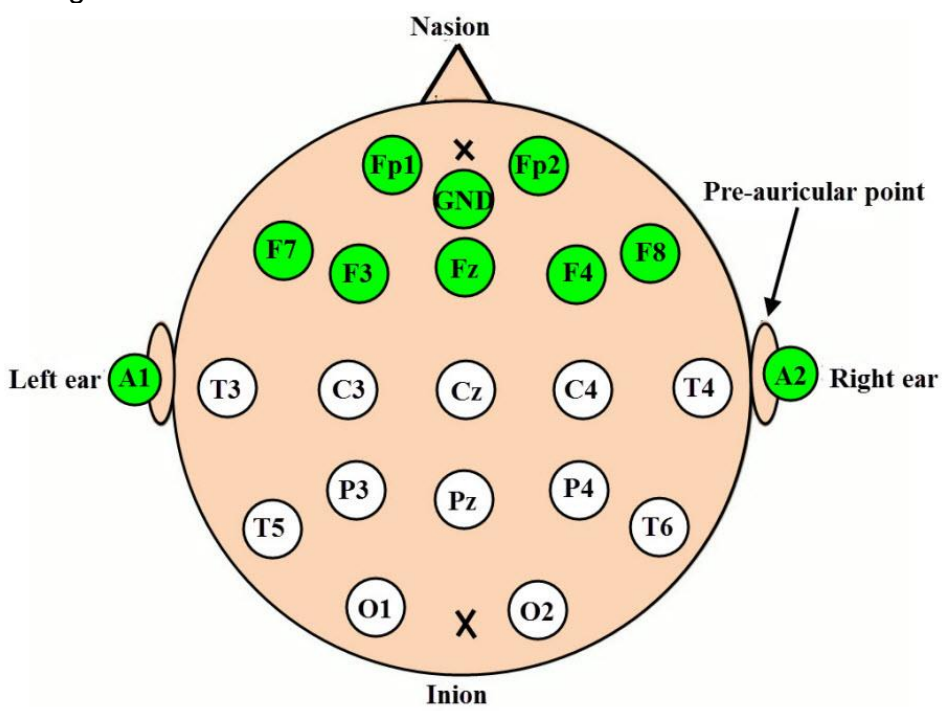

Fig. 3 The placement of electrodes

In the following section, we firstly analyze two typical patients' EEG that are from different patients, respectively one coma patient and one quasi-brain-death patient, by Dynamic 2T-EMD. Secondly, we consentrate on the special patient who was from coma to quasi-brain-death. Finally, we summary the average EEG energy of 6 channels in the whole recording time period for 36 cases of patients' EEG by Dynamic 2T-EMD.

\subsection{Results}

\subsubsection{Result analysis of one coma patient's EEG and one quasi-brain-death patient's EEG}

We analyze one coma patient's EEG with a record duration of 909 s and one quasi-brain-death patient's EEG with a record duration of 1088s. As is shown in Figure 4 and Figure 5. We select EEG energy curve for the first 50 s to observe. The results illstrate that the dynamic EEG energy of each channel for coma patient's EEG is higher than $1 \times 10^{4}$, and the variation range is $1.71 \times 10^{4} \sim 1.98 \times 10^{5}$, while the dynamic EEG energy for quasi-brain-death patient's EEG is far lower than $1 \times 10^{4}$ with changing range from $1.23 \times 10^{3} \sim 6.06 \times 10^{3}$. Then we compute the average dynamic EEG energy of 6 channels for the two cases, as is shown in Figure 6 and Figure 7, and the average dynamic EEG energy of coma patient's EEG is obviously higher than that of quasi-brain-death at any time.

According to the definition of EEG energy, the higher energy indicates that there are obvious periodic rhythms in patient's EEG, that is to say, the patient has brain activity in the coma state. While quasi-brain-death patient has almost no brain activity, and as there exists random noise in EEG, there is lower EEG energy in quasi-brain-death patient. 


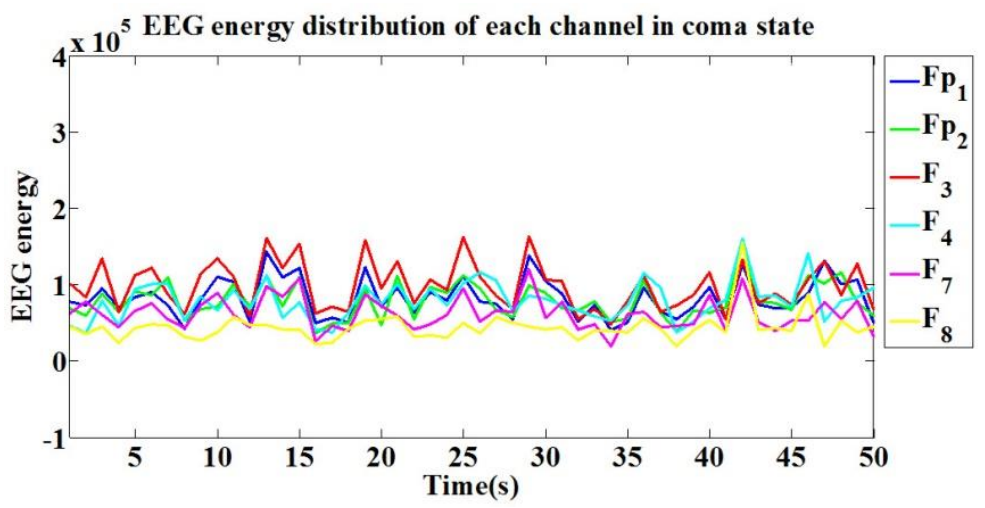

Fig. 4 EEG energy distribution of each channel in coma state

EEG energy distribution of each channel in quasi-brain-death state

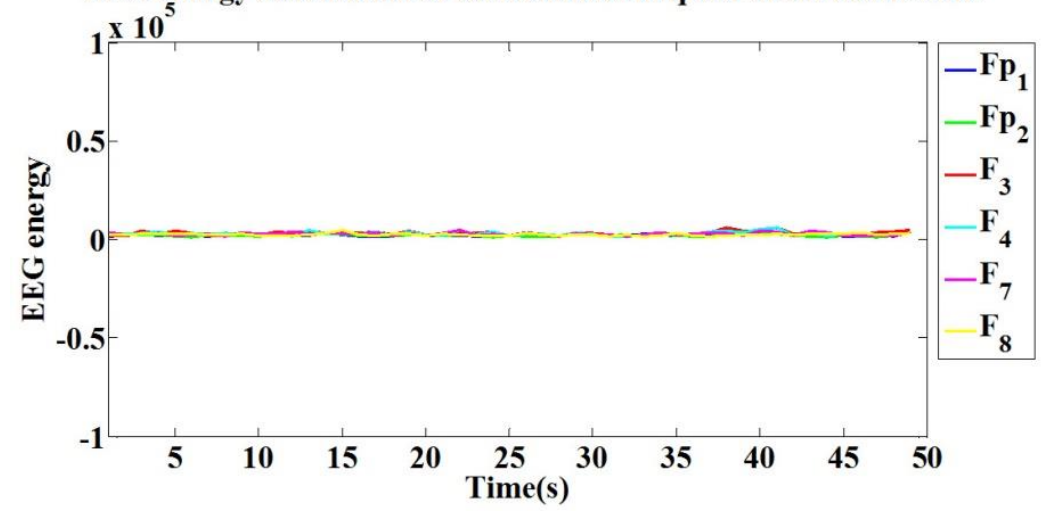

Fig. 5 EEG energy distribution of each channel in quasi-brain-death state

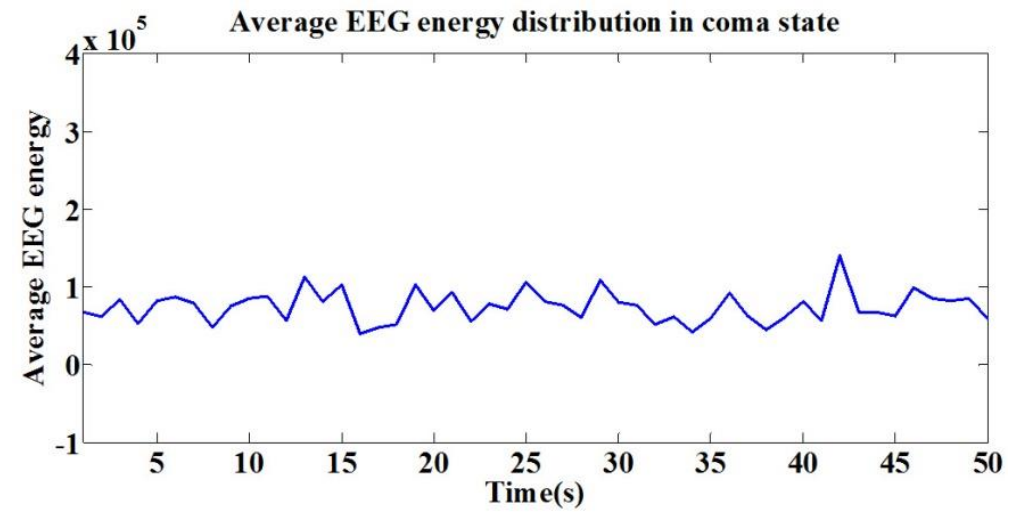

Fig. 6 Average EEG energy distribution in coma state

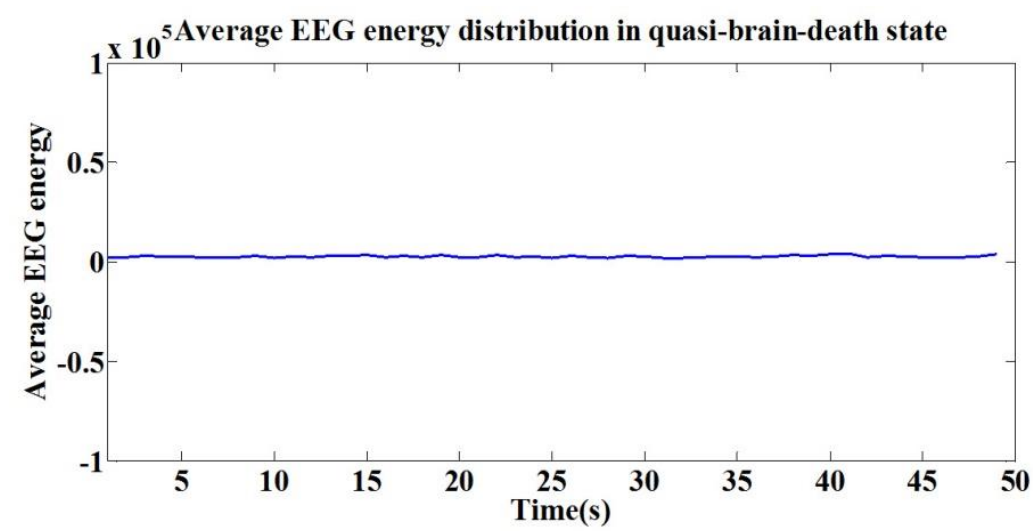

Fig. 7 Average EEG energy distribution in quasi-brain-death state 


\subsubsection{Result analysis of the patient's EEG from coma to quasi-brain-death state}

Then we process the patient's EEG from coma to quasi-brain-death state. The patient lost cognitive and motor functions, the pupil dilated to $4 \mathrm{~mm}$ that only had a weak visual response, and a respiratory machine was used. And the patient lost consciousness unexpectedly some time in October 2005. The first EEG examination was taken with a record duration of 900 s, and the EEG recorded was in coma state. After about 10h on the same day, the patient's condition appeared worse and was found to have completely lost reaction to external visual, auditory and tactile simulation. Then the diagnosis was made as a quasi-brain-death case by two physicians. The EEG examination was taken for the second time, and the recorded time is $1153 \mathrm{~s}$ (Q. Shi et al., 2011). We select the last 50 s of EEG in coma state and the beginning 50 s of EEG in quasi-brain-death to analyze.

As is shown in Figure 8 and Figure 9, the analysis results illustrate intuitively that EEG energy trend from coma to quasibrian-death state. In the state of coma, the average EEG energy varies within the range of $2.02 \times 10^{4} \sim 5.59 \times 10^{4}$, while in the state of quasi-brain-death the EEG energy is varies in the range of $2.26 \times 10^{3} \sim 4.82 \times 10^{3}$, lower than $1 \times 10^{4}$. EEG energy in coma state is obviously higher than that in quasi-brain-death state in the whole dynamic EEG energy distribution curve.

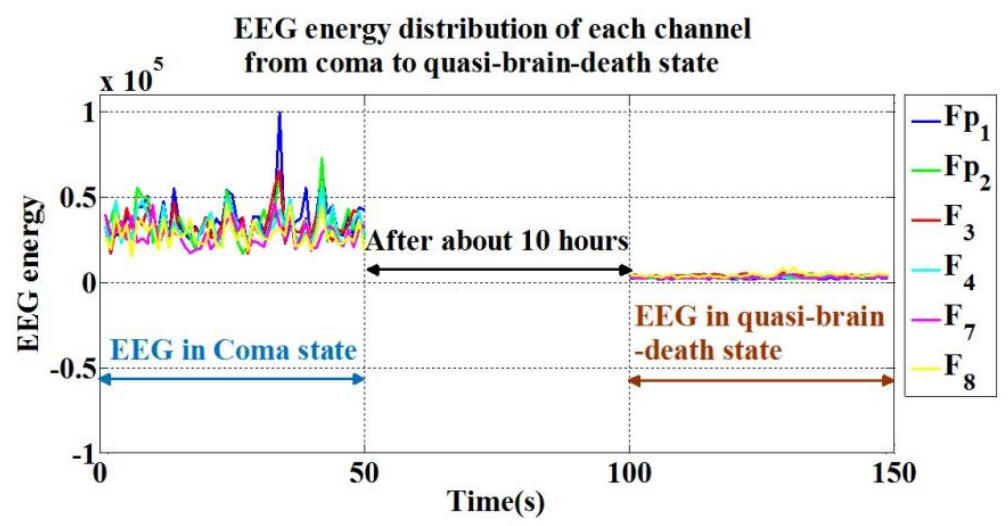

Fig. 8 EEG energy distribution of each channel from coma to quasi-brain-death state

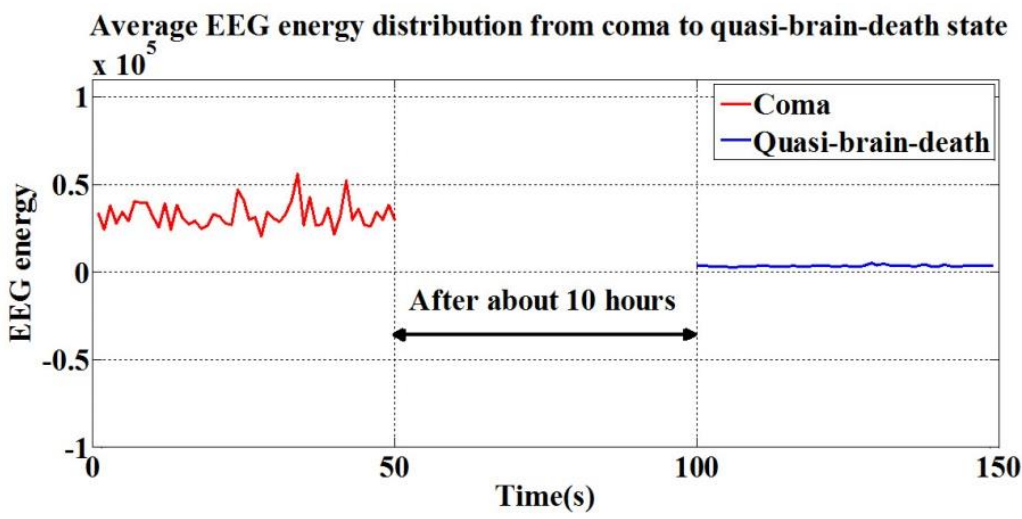

Fig. 9 Average EEG energy distribution in quasi-brain-death state

\subsubsection{Summary of average EEG energy of patients' EEG}

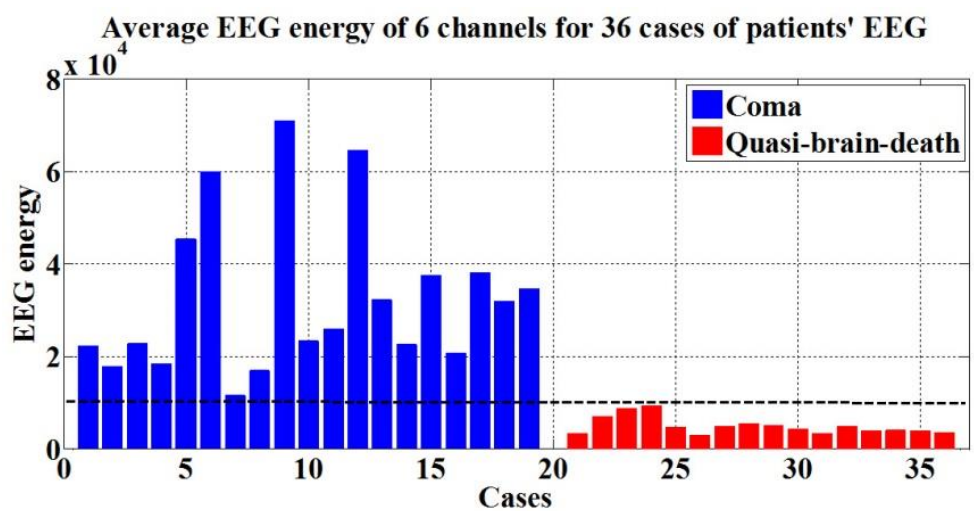

Fig. 10 Average EEG energy of 6 channels for 36 cases of patients' EEG 
We apply Dynamic 2T-EMD to obtain the average EEG energy of 6 channels in the entire recording time for all 36 cases of patients' EEG, which can prevent the information loss and wrong static results caused by some artificial factors or interference noises of equipment in EEG examination. As is shown in Figure 10, it is obviously shown that the average EEG energy of coma patients' EEG is higher than $1 \times 10^{4}$, and it indicates that there exists brain activity. While the average EEG energy of quasi-brian-death patients' EEG is lower than $1 \times 10^{4}$, which explain there almost no brain activity apart from some kinds of noises.

\section{CONCLUSION}

In this paper, we have proposed the Dynamic 2T-EMD by extending 2T-EMD to analyze dynamically EEG energy. It's the algorithm by introducing two parameters of the time window $\Delta t$ and the time step $\Delta \lambda$. With the time window sliding along the time axis, the EEG in the corresponding time step is analyzed and the EEG energy is computed. Then we apply Dynamic 2T-EMD to analyze 36 cases of coma and quasi-brain-death patients' EEG. We showed two examples for two typical patients' EEG including one coma patient's EEG and one quasi-brain-death patient's EEG, and a special paitent's EEG that the patient was from coma state to quasi-brain-death state to analyze by Dynamic 2T-EMD, and finally we summary average EEG energy of 36 cases of patients' EEG for corresponding recording time. The results present dynamic EEG energy distribution in coma and quasi-brain-death state, and also illustrate that EEG energy in coma state is higer than that in quasi-brain-death state from perspective of dynamic EEG energy. And the results can prevent information loss and wrong static EEG energy resulted by noise interference and provide doctors scientific basis to evaluate patients' conciousness level. More importantly, The proposed algorithm is extremely important to the real time brain death diagnostic system.

In the future work, we will focus on developing the real time brain death diagnostic system by connecting the EEG measurement system to the EEG analysis system to realize real time analysis of EEG.

\section{ACKNOWLEDGMENTS}

This work was partly supported by KAKENHI (25420417).

\section{REFERENCES}

1. Becheer, H. K., et al.: "Report of the Ad hoc committeeof the Harvard medical school to examine the definition of brain death: A definition of irreversibal coma", JAMA, Vol. 205, No. 6, pp.337-340, 1968.

2. Wijdicks EFM: "Brain death worldwide: accept fact but no global concensus in diagnostic criteria", Neurology, Vol. 58, No. 1, pp.20-25, 2002.

3. A. Hyvarien, E. Oja:"A fast fixed-point algorithm for independent component analysis", Neural Computation, Vol. 9, No. 7, pp.1483-1492, 1997.

4. L. Li, Y. Saito, D. Looney, et al.: "Data fusion via fission for the analysis of brain death", Evolving Intelligence Systems: Methodology and Applications, Springer, pp.279-320, 2008.

5. Gennady G. Knyazev, Jaroslav Y. Slobodskoj-Plusnin, Andrey V. Bocharov, et al.:"The default mode network and EEG alpha oscillations: an independent component analysis", Brain research, Vol. 1402, pp.67-69, 2011.

6. J. Cao, N. Murata, S. Amari, A. Cichocki and T. Takeda: "A robust approach to independent component analysis of signal with high-level noise measurement", IEEE Trans Neural Netw, Vol. 16, No. 3, pp.631-645, 2003.

7. N. Huang, Z. Shen, S. Long, M. Wu, H. Shih, Q. Zheng, N. Yen, C. Tung, and H. Liu: "The empirical mode decomposition and Hilbert spectrum for non-linear and non-stationary time series analysis", Proceedings of the Royal Society of London, A 454, pp.903-995, 1998.

8. N. Rehman and D. Mandic: "Multivariate empirical mode decomposition", Proceedings of the Royal Society A, Vol. 466, No. 2117, pp.1291-1302, 2010.

9. Julien Fleureau, Jean-Claude Nunes, Amar Kachenoura, Laurent Albera, and Lotfi Senhadji: "Turning Tangent Empirical Mode Decomposition: A Framework for Mono- and Multivariate Signals", IEEE Trans Signal Process, Vol. 59, No. 3, pp.1309-1316, 2011.

10. Z. Chen, J. Cao, Y. Cao, et al: "Qualitative evaluation and quantitative EEG analysis in brain death diagnosis for adults: An empirical study", Cognitive Neurodynamics, Springer, Vol. 2, No. 3, pp.257-271, 2008.

11. E. Niedermeyer, et al: "Coma and brain death", In Electroencephalograph: basic principles, clinical applications, and related fields, Chapter 26, Lippincott Williams \& Wilkins, Baltimore, MD, 1991.

12. T. M. Rutkowski, D. P. Mandic, A. Cichocki, et al: "EMD approach to multichannel EEG data-the amplitude and phase components clustering analysis", Journal of Circuits, Systems, and Computers, Vol. 19, No. 1, pp.215-229, 2010.

13. Q. Shi, J. Cao, T. Tanaka, R. Wang, and H. Zhu.: "EEG data analysis based on EMD for coma and quasi-braindeath patient", Journal of Experimental and Theoretical Artificial Intelligence, Vol. 23, No. 1, pp.97-110, 2011. 
14. Julien Fleureaua, Amar Kachenouraa, Laurent Alberaa, Jean-Claude Nunesa, and Lotfi Senhadjia: "Multivariate empirical mode decomposition and application to multichannel filtering", Signal Processing, Vol. 91, No. 12, pp.2783-2792, 2011.

15. J. Cao: "Analysis of the quasi-brain-death EEG data based on a robust ICA approach", Lecture Notes in Artificial Intelligence, Springer, Vol. 4253, No. 3, pp.1240-1247, 2006.

\section{Author' biography with Photo}

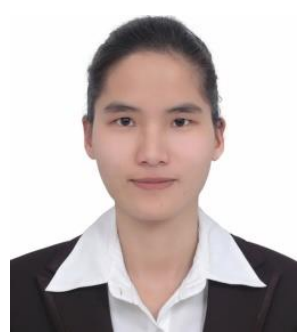

Yao Miao, Ph. D. student, will received Ph.D. degree in signal processing, information science, Saitama Institute of Technology, Japan, in 2019. Her research topics are EEG signal processing, algorithms and system on EEG preliminary examination for Brain Death Determination. She has published several papers and attended several conference to give presentations of EEG signal processing. She is a member of IEEE and IEEE Computational Intelligence Society.

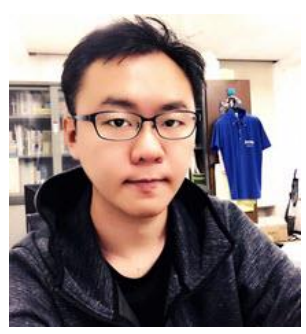

Dongsheng Wang, Master student, will received Master degree in 2017. He has attended several conferences. His research topics are computer programming language and large-scale signal processing.

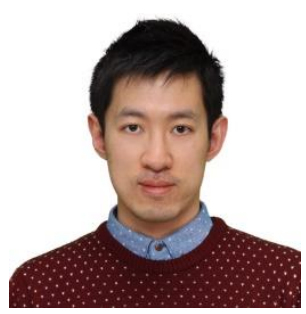

Gaochao Cui, Ph.D. student, will received Ph. D. degree in signal processing, information science, Saitama Institute of Technology, Japan, in 2017. He has published several papers related signal processing and brain computer interface. Many conferences have invited him to give presentations to the international researchers. In the first year of Ph. D., he was selected as the member of Junior Research Associate Program in REKEN, Japan and worked out ICA toolbox with other colleagues.

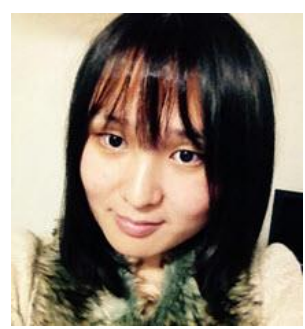

Li Zhu, Ph.D. student, will receive Ph.D. degree in computer science. Cognitive Science, Xiamen University. Her research topics are related to data mining, signal processing and cloud computing. She has published several papers, one book and patent related to power system modeling, machine learning and big data processing.

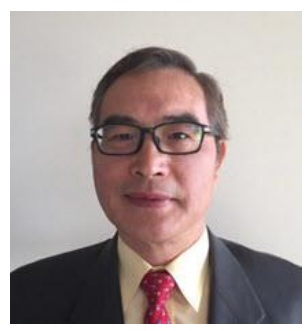

Jianting Cao, received the M. Eng. and Ph.D. degrees from the Graduate School of Science and Technology, Chiba University, Japan, in 1993 and 1996, respectively. From 1983 to 1988, he worked as a Researcher at the Institute of Technology and Equipment in the Ministry of Geological and Mineral in China. From 1996 to 1998, he worked as a Researcher at the Brain Science Institute, RIKEN (The Institute of Physical and Chemical Research) in Japan. From 1998 to 2002, he worked as an Assistant, and a Lecture at the Sophia University in Japan. From 2002 to 2007, he worked as an Associate Professor at the Saitama Institute of Technology in Japan. $\mathrm{He}$ is currently working as a Professor at the Department of Information Systems, Saitama Institute of Technology, and a Visiting Research Scientist at the Brain Science Institute, RIKEN in Japan. He received the Best Paper Award from the Telecommunications Advancement Foundation (Japan) in 1996, from the IEEE Circuits and System Society in 2005, from the Signal Processing Institute (Japan) in 2007, and from International Neural Network Society in 2010. His research interests include blind signal processing, biomedical signal processing, neural networks and learning algorithms. Dr. Cao is a member of IEEE, and IEICE (Japan). Corresponding author.

7580 | P a g e 